\title{
On the Vilsmeier formylation of $N$-aryl-substituted 2-aminothiophenes - a simple route to new thieno[2,3-b]quinolinium salts
}

\author{
Horst Hartmann* \\ Department Chemie, Technische Universität Dresden, Dresden, Germany \\ E-mail: Hartmann@iapp.de
}

Dedicated to Prof. Dr. Rainer Beckert on the occasion his $60^{\text {th }}$ birthday

\begin{abstract}
The Vilsmeier reaction of $N$-substituted 2-arylamino-thiophenes-5-carboxylic acid or their alkyl derivatives gives rise, depending on the substitution pattern at the thiophene moiety, to the formation of either $\mathrm{N}$-substituted 2-arylamino-thiophene-5-carbaldehydes, corresponding imminium salt precursors, or novel thieno[2,3-b]quinolinium salts. These salts are highly reactive towards nucleophiles and can be easily transformed by reduction into the corresponding 4,9-dihydro derivatives.
\end{abstract}

Keywords: aromatic substitution, heterocycles, Vilsmeier formylation, reduction, 2aminothiophenes, cations

\section{Introduction}

Similarly to $N, N$-disubstituted anilines the heterocyclic $N, N$-disubstituted 2-aminothiophene analogues 1 are highly reactive towards a large variety of electrophilic reagents. As far as the 5position in these electron-rich heterocyclic compounds $\mathbf{1}$ is unsubstituted, 5-substituted 2aminothiophene derivatives $\mathbf{2}$ are formed by reaction with these reagents. For instance, with the Vilsmeier reagent (VR), ${ }^{1}$ with nitrous acid and its derivatives, ${ }^{2}$ or with aryldiazonium salts ${ }^{3}$ the corresponding substitution products $\mathbf{2 a}, \mathbf{2 c}$ and $\mathbf{2 d}$, respectively, are formed (Scheme 1). All these compounds received a lot of interest as versatile starting materials for deeply coloured methine, azamethine and azo dyes, some of which exhibit interested non-linear optical properties which enable to use these compounds for opto-electronic applications, eg. as NLO materials, ${ }^{4}$ as NIR dyes, ${ }^{5}$ or as sensors for estimating the solvent polarity. ${ }^{6}$

Furthermore, $N, N$-disubstituted 2-aminothiophenes $\mathbf{1}$ have been used, as far as they are $N$ aryl-substituted, as building blocks for a variety of oligomeric compounds of the general 
structure $\mathbf{3}_{\mathbf{n}},{ }^{7} \mathbf{4}_{\mathbf{n}}{ }^{8}$ and $\mathbf{5}_{\mathbf{n}},{ }^{8}$ useful e.g. as hole transport materials for manufacturing optoelectronic devices. ${ }^{10}$
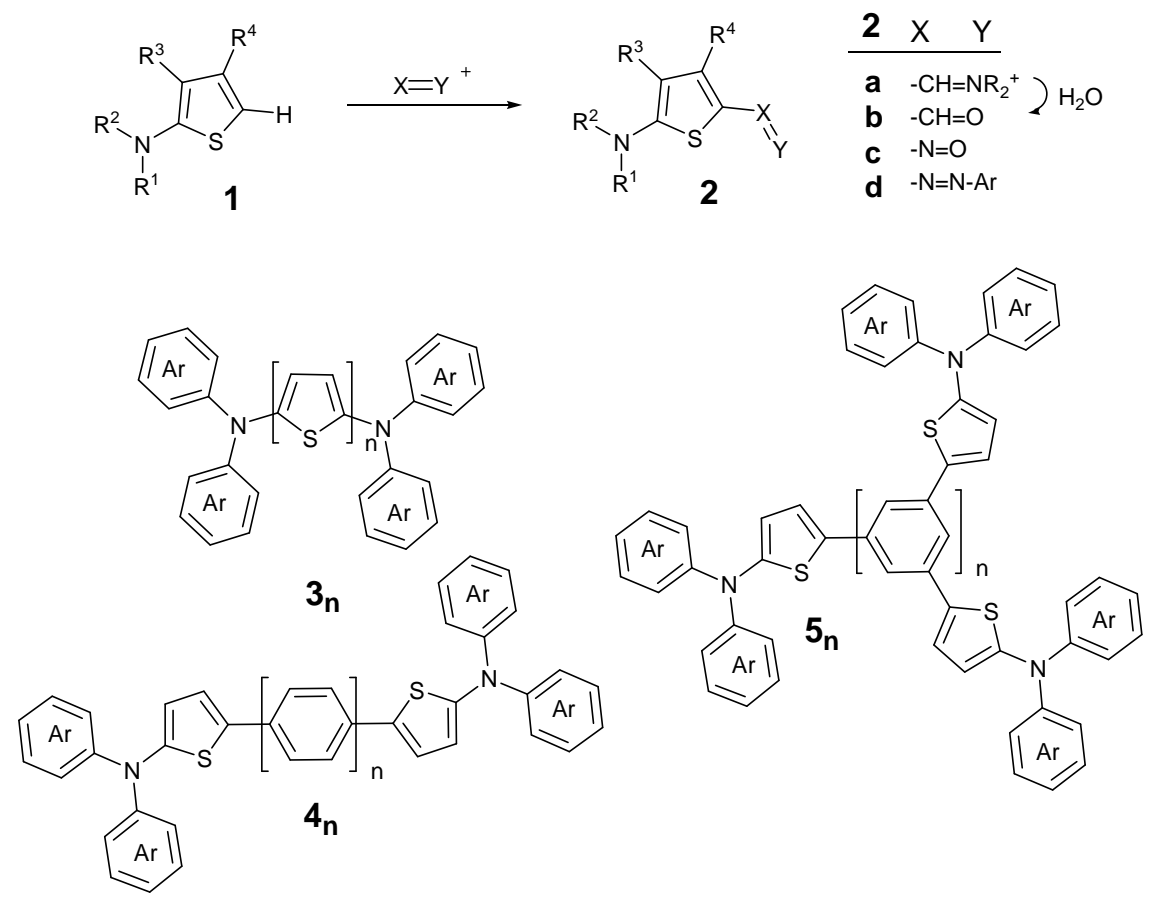

\section{Scheme 1}

Obviously, a simple access to the $N, N$-disubtituted 2-aminothiophenes $\mathbf{1}$ was an important precondition for the preparation and application of the before-mentioned compounds. This access was found in a heavy-metal catalysed $C$ - $N$-coupling of 2-halothiophenes 6 with secondary amines (Scheme 2). ${ }^{11}$ This route allowed to prepare a large variety of $N, N$-disubtituted 2aminothiophenes 7 but it gives in several cases only low yields of products. ${ }^{12}$ Therefore, another synthetic route to the target compounds was an advantageous alternative. It was developed by us recently and consists in a heterocyclisation reaction starting from simple $N, N$-disubtituted acetamides 11. ${ }^{13}$ These compounds can be simply transformed, via the corresponding $N, N$ 'tetrasubstituted 1-amino-1-chloro-propeniminium salts $\mathbf{1 0}, \quad$ into $N, N$-disubtituted 2aminothiophene-carboxylic esters $\mathbf{9}$ and acids $\mathbf{8}$ from which the target compounds $\mathbf{7}$ are available by a simple decarboxylation reaction. This heterocyclisation route allows to prepare differently $N, N$-disubstituted 2-aminothiophenes in satisfactory yields mostly and opens the door for further synthetic transformations. ${ }^{14}$ 


\section{Results and Discussion}

Continuing our efforts for using $N, N$-disubstituted 2-aminothiophenes and their derivatives as starting materials for a variety of new compounds with interesting chemical and physical properties we studied the reaction of $N$-aryl-substituted 2 -aminothiophenes $7\left(\mathrm{R}^{1}\right.$ and/or $\mathrm{R}^{2}=$ aryl) as well as their corresponding 5-carboxylic acids $\mathbf{8}$ and methyl carboxylates $\mathbf{9}$ towards the VR. In course of this study we found some interesting results which parallel, in general, with the reactivity of $N, N$-dialkyl-substituted 2 -amino-thiophenes $7\left(\mathrm{R}^{1} / \mathrm{R}^{2}=\right.$ Alkyl) but differ in some cases from the one of the $N, N$-dialkyl-substututed compounds with this reagent. Thus, by starting from $N$-phenyl-substituted 5-aminothiophenes $7\left(\mathrm{R}^{1}=\right.$ Aryl) as expected iminium salts 12 were obtained as primary reaction products. ${ }^{1}$ The same type of salts $\mathbf{1 2}$ were obtained by starting with the $N, N$-dialkyl-substiuted analogues and could be transformed by hydrolysis into the corresponding formyl derivatives 13. Similar results have been obtained by starting from $N, N$ disubstituted 5-aminothiophene-2-carboxylic acids $\mathbf{8}$. With the VR both the $N$-alkyl- as well as $\mathrm{N}$-aryl-substituted compounds $\mathbf{8}$ were transformed into the iminium salts $\mathbf{1 2}$ or formyl derivatives 13 indicating that decarboxylation of the acids, used in course of the formylation reaction, had occured.

The appropriate iminium salts $\mathbf{1 2}$ could be easily isolated, e.g. as perchlorates, after quenching the reaction mixture with methanol by addition of perchloric acid and the formyl derivatives 13 were obtained by neutralisation of the reaction mixture with aqueous sodium hydroxide.

By starting with $\mathrm{N}$-aryl-substituted 5-aminothiophene-2-carboxylic esters $\mathbf{9}$, however, neither corresponding 4-formyl-derivatives $\mathbf{1 7}$ or the iminium salt precursors $\mathbf{1 6}$ nor corresponding 4'formyl derivatives $\mathbf{1 5}$ or imimium salts $\mathbf{1 4}$ bearing the $\mathrm{CH}=\mathrm{X}$ group at the aryl moiety of the starting esters were formed. Instead, 2-methoxycarbonyl-substituted thieno[2,3-b]quinolinium salts 18 were obtained. These salts could be conveniently isolated in satisfactory yields as pale yellow coloured perchlorates after addition of perchloric acid to the reaction mixture quenched with methanol. Obviously, the starting $N$-aryl-substituted methyl carboxylates 9 were transformed in the first step into the corresponding iminium salts $\mathbf{1 6}$ which cyclize subsequently under the reaction conditions applied to yield the heterocyclic products $\mathbf{1 8}$.

The heterocyclisation reaction found has some analogies, as would be worth mentioning, in the reaction of $N$-substituted 2-( $N$-phenyl)-naphthylamines with the VR which transform the starting compounds into appropriate benz[a] acridinium salts. ${ }^{15}$

The structures of 12, 13, 16 and 17, as well as of the thieno[2,3-b]quinolinium perchlorats 18 prepared follow unambiguously from their analytic data recorded. Thus, in the ${ }^{1} \mathrm{H}$ NMR spectra of the iminium salts 12a, 12b and 12e, prepared as examples from the corresponding $N, N$ disubstituted 2-aminothiophene derivatives 7 and $\mathbf{8}$, characteristic singlets between $7.8-8.7 \mathrm{ppm}$ and a couple of singlets at 3.3 and $3.5 \mathrm{ppm}$ were recorded in addition to a series of multiplets. The singlets can be attributed to the protons at the iminium moieties and $N$-methyl groups, respectively, whereas the multiplets can be attributed to the protons at the aryl moieties. In the ${ }^{1} \mathrm{H}$ 
NMR spectra of the formyl derivatives $13 \mathbf{a}, 13 \mathbf{b}, \mathbf{1 7} \mathbf{a}$, and $\mathbf{1 7} \mathbf{b}$, prepared from the corresponding iminium salt precursors 12 or directly from the $N, N$-disubstituted methyl 2-aminothiophene carboxylates 9, respectively, characteristic singlets between 8.7 and $9.5 \mathrm{ppm}$ originated from the protons at the formyl groups and at about $7.9 \mathrm{ppm}$ originated from the protons at the thiophene moieties in the compounds $\mathbf{1 7} \mathbf{a}$ and $\mathbf{1 7} \mathbf{b}$ were recorded. Additionally, characteristic doublets in the ${ }^{1} \mathrm{H}$ NMR spectra of the imminium salts 12 at about $6.4 \mathrm{ppm}$ and between $7.5-7.9 \mathrm{ppm}$ with coupling constants of $4.3-4.8 \mathrm{~Hz}$ originated from the protons at the thiophene moieties were observed.

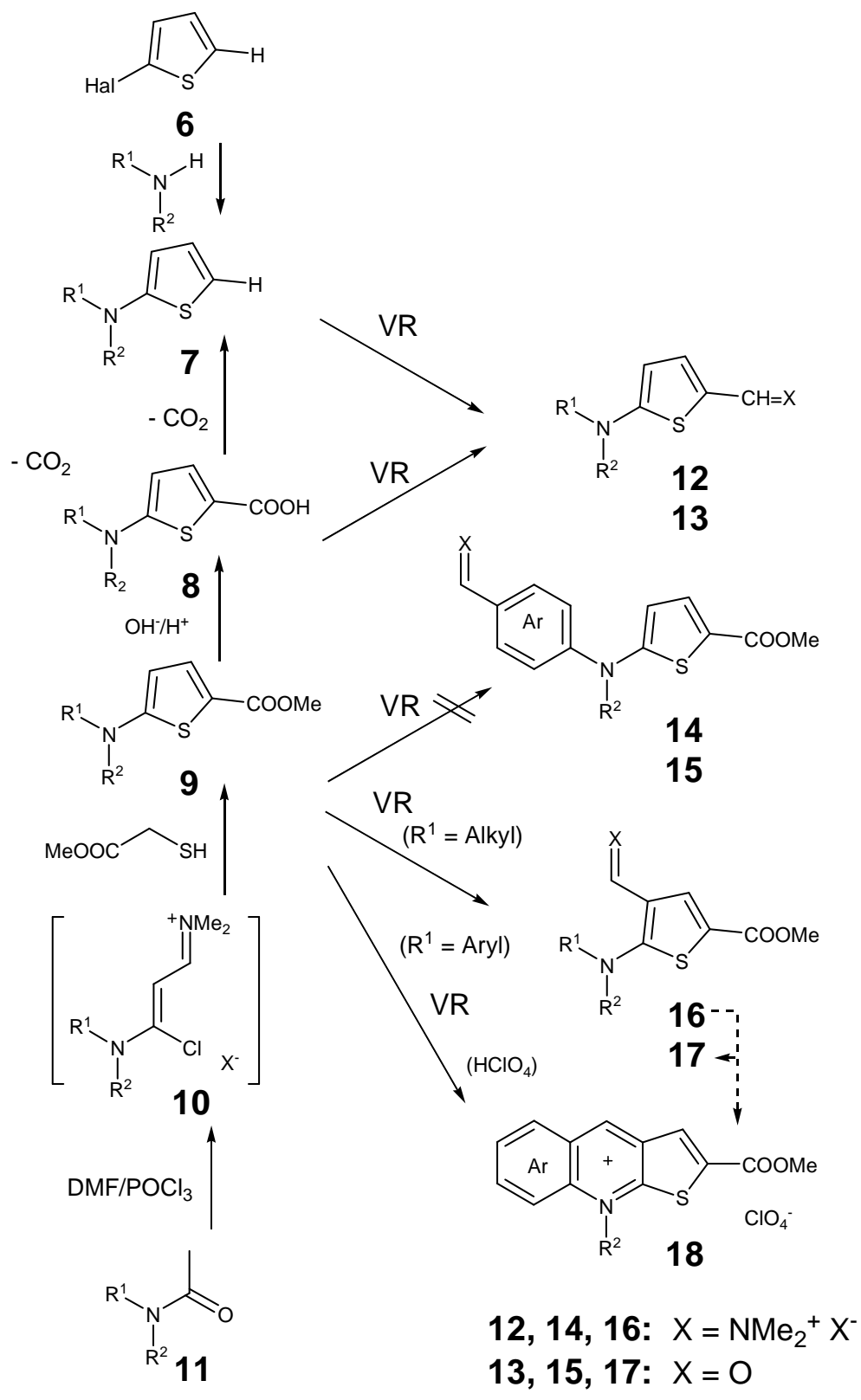

Scheme 2 
In the ${ }^{1} \mathrm{H}$ NMR spectra of the thieno[2,3-b]quinolinium salts $\mathbf{1 8}$, besides several multiplets between 7.0 and $9.5 \mathrm{ppm}$ corresponding to the protons of the aryl moieties, characteristic singlets at about 4.0, 8.7 and $10.0 \mathrm{ppm}$ were recorded. These signals can be attributed to the methyl group at the carboxylate moieties and to the protons at the 3- and 4-positions of the heterocyclic thieno[2,3- $b$ ]quinolinium moieties, respectively.

First attempts to transform the new thieno[2,3-b]quinolinium salts 18 into certain consecutive products demonstrate that these salts are able to react with a variety of nucleophiles, such as with aliphatic amines, sulfides or with $N, N$-disubstituted aromatic or heteroaromatic amines. Furthermore, they are able to react with usual reducing agents. For instance, by heating the thieno[2,3-b]quinolinium perchlorate 18 with 2-morpholino-thiophene-5-carboxylic acid $\mathbf{8 j}$ in acetic anhydride a deeply coloured condensation product of structure $\mathbf{1 9}$ was obtained (Scheme $3)$. In accordance with the analytical data of the product we assume the corresponding addition products is formed first and then subsequently transformed by hydride abstraction into the target products 19 by air oxygen or by using a second equivalent of the starting thieno[2,3$b$ ]quinolinium salt 18.

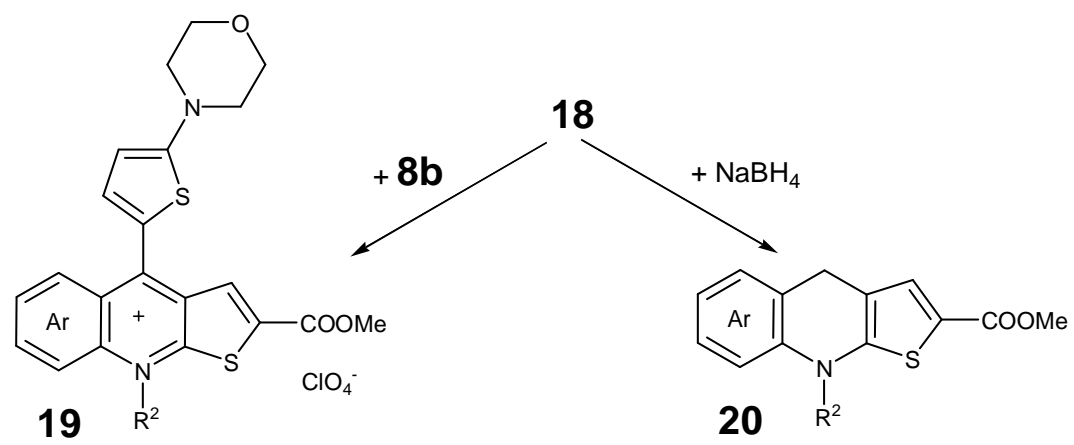

\section{Scheme 3}

By allowing to react the thieno[2,3- $b$ ]quinolinium perchlorates $\mathbf{1 8}$ with sodium borotetrahydride in methanol or DMF the corresponding 4,9-dihydro-thieno[2,3-b] quinolines 20 were formed. In contrast to the salts 19, these compounds exhibit in their ${ }^{1} \mathrm{H}$ NMR spectra characteristic signals at about $4.6 \mathrm{ppm}$ for the protons at the $\mathrm{CH}_{2}$ group. The 4,9-dihydrothieno[2,3- $b$ ]quinolines $\mathbf{2 0}$ are nearly colourless compounds and exhibit intense fluorescence in the blue spectral region. With strong electron accepting compounds the 4,9-dihydro-thieno[2,3$b$ ]quinolines 20 can act as hydride donors. Detailed information on this characteristic property will be reported shortly. 


\section{Experimental Section}

General. ${ }^{1} \mathrm{H}$ NMR and ${ }^{13} \mathrm{C}$ NMR spectra were recorded with a Bruker DRX $500 \mathrm{P}$ instrument at 500.13 and $125.76 \mathrm{MHz}$, respectively. Elemental analyses were performed with a Eurovektor Hekatech EA-3000 elemental analyzer. The mass spectra were recorded with a Bruker EsquireLC 00084 instrument and the melting points with a Boetius heating-table microscope.

The $N, N$-disubstituted 2-aminothiophenes 7 as well as the $N, N$-disubstituted 5-aminothiophene2-carboxylic acids $\mathbf{8}$ and their methyl derivatives $\mathbf{9}$ used as starting materials were prepared accordingly to the literature. ${ }^{13 a, 16}$. The following starting materials not described in detail hitherto were prepared according to the literature by reaction of the corresponding 1-chloropropene-3-(N,N-dimethyl)-iminium salt with methyl thioglycolate. ${ }^{13}$

\section{General procedure for preparation of $N$-disubstituted methyl 2-aminothiophene-5-} carboxylates (9)

(a) Preparation of $N, N$ '-tetrasubstituted 1-amino-1-chloro-propeniminium salts (10). To a solution of $N, N$-disubstituted acetamide $(0.1 \mathrm{~mol})$ in DMF $(25 \mathrm{~mL}) \mathrm{POCl}_{3}(30.0 \mathrm{~g}, 0.2 \mathrm{~mol})$ was added under stirring and cooling. The resulting mixture was left standing over night at room temperature and then poured in ice water $(250 \mathrm{~mL})$. After addition of perchloric acid $(70 \%, 25$ $\mathrm{mL})$ the aqueous solution was extracted with dichloromethane $(2 \mathrm{x} 200 \mathrm{~mL})$ and the organic phase was evaporated after drying with $\mathrm{MgSO}_{4}$. The residues crystallised after some standing and have been used without further purification for the next step.

(b) Preparation of $\mathrm{N}, \mathrm{N}$-disubstituted methyl 2-aminothiophene-5-carboxylates (9). To a mixture of a $N, N$ '-tetrasubstituted 1-amino-1-chloro-propeneiminium perchlorate $\mathbf{1 0}(0.1 \mathrm{~mol})$ and methyl thioglycolate $(12.0 \mathrm{~g}, 0.11 \mathrm{~mol})$ in acetonitrile $(100 \mathrm{~mL})$ sodium methanolate was added under stirring at room temperature until the mixture attains a $\mathrm{pH}>9$. After some standing, the resulting mixture was diluted with water $(500 \mathrm{~mL})$ until the products start to crystallise. They were isolated by suction and recrystallised for purification from toluene.

The following $N, N$-disubstituted methyl 2-aminothiophene-5-carboxylates 9 not described in the literature hitherto were so obtained:

Methyl 5-[( $N$-(1-naphtyl)- $N$-phenyl]amino-thiophene-2-carboxylate

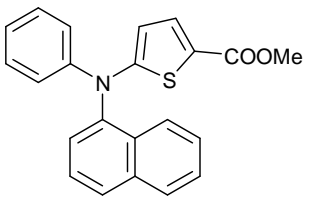

(9b) from $N$-(1-naphthyl)- $N$-phenyl-acetamide ${ }^{17}$ (11b) via the corresponding 1-chloro-3-dimethylamino-1- $N$-(1-naphthyl)- $N$-phenyl)propeniminium perchlorate $(\mathbf{1 0 b})$ in a yield of $85 \%$ and a m.p. of 90 $91^{\circ} \mathrm{C} .{ }^{1} \mathrm{H}$ NMR $\left(500 \mathrm{MHz}, \mathrm{CDCl}_{3}\right): \delta=3.79\left(\mathrm{~s}, 3 \mathrm{H}, \mathrm{OCH}_{3}\right), 6.35(\mathrm{~d}, J=$ $\left.5.6 \mathrm{~Hz}, \mathrm{CH}_{\mathrm{th}}\right), 7.05-7.09$ (m, 1 arom. H), $7.26-7.28$ (m, 4 arom. H), $7.45-7.53$ (m, 5 arom. $\mathrm{H}), 7.54\left(\mathrm{~d}, J=5.6 \mathrm{~Hz}, \mathrm{CH}_{\mathrm{th}}\right), 7.86-8.00(\mathrm{~m}, 2$ arom. $\mathrm{H}) ;{ }^{13} \mathrm{C} \mathrm{NMR}\left(125.76 \mathrm{MHz}, \mathrm{CDCl}_{3}\right): \delta=$ $51.46,115.46,121.39,121.52,123.70,124.58,125.01,125.50,126.60,127.33,127.63,129.44$, $129.60,130.93,133.81,134.03,144.28,145.80,159.66,162.83$.

Anal. Calcd for $\mathrm{C}_{22} \mathrm{H}_{17} \mathrm{NO}_{2} \mathrm{~S}$ (359.44): C 73.51, H 4.77, N 3.90; found C 73.66, H 4.71, N 3.83. 


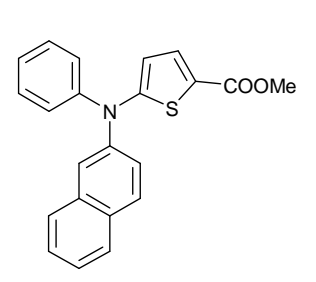

Methyl 5-[( $N$-(2-naphtyl)- $N$-phenyl] amino-thiophene-2-carboxylate (9c), from $N$-(2-naphthyl)- $N$-phenyl-acetamide ${ }^{17}$ (11c) via the corresponding 1-chloro-3-dimethylamino-1- $N$-(2-naphthyl)- $N$-phenyl)propeniminium perchlorate $(\mathbf{1 0 c})$ in a yield of $85 \%$ and a m.p. of $117-$ $118{ }^{\circ} \mathrm{C} .{ }^{1} \mathrm{H}$ NMR $\left(500 \mathrm{MHz}, \mathrm{CDCl}_{3}\right): \delta=3.82\left(\mathrm{~s}, 3 \mathrm{H}, \mathrm{OCH}_{3}\right), 6.50(\mathrm{~d}, J=$ $\left.5.6 \mathrm{~Hz}, 1 \mathrm{H}, \mathrm{CH}_{\mathrm{th}}\right), 7.17(\mathrm{~m}, 1$ arom. $\mathrm{H}), 7.26(\mathrm{t}, J=2.0 \mathrm{~Hz}, 1$ arom. $\mathrm{H})$, $7.28(\mathrm{t}, J=2.0 \mathrm{~Hz}, 1$ arom $\mathrm{H}), 7.32-7.37$ (m, 3 arom. H), $7.40-7.48$ (m, 2 arom. H), 7.59 (d, $J$ $\left.=5.6 \mathrm{~Hz}, 1 \mathrm{H}, \mathrm{CH}_{\mathrm{th}}\right), 7.63-7.69(\mathrm{~m}, 2$ arom. $\mathrm{H}), 7.78-7.80(\mathrm{~m}, 2$ arom. $\mathrm{H}) ;{ }^{13} \mathrm{C} \mathrm{NMR}(125.76$ $\left.\mathrm{MHz}, \mathrm{CDCl}_{3}\right): \delta=51.79,112.79,119.90,121.62,123.26,123.74,126.19,126.50,126.80$, 127.08, 128.20, 128.54, 129.35, 130.47, 134.00, 135.21, 142.33, 147.38, 160.60, 162.27 .

Anal. Calcd for $\mathrm{C}_{22} \mathrm{H}_{17} \mathrm{NO}_{2} \mathrm{~S}$ (359.44): C 73.51, H 4.77, N 3.90; found C 73.38, H 4.81, N 4.02

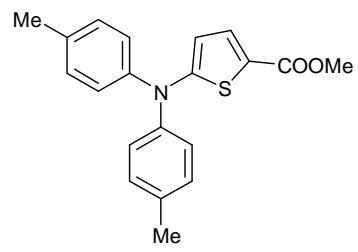

Methyl 5-(di-4-tolyl-amino)-thiophene-2-carboxylate (9d), from $N, N$-bis-(4-tolyl)-acetamide (11d) [18] via the corresponding 1chloro-1-[bis-(4-tolyl)amino]-propene-3-dimethylmethiniminium perchlorate (10d) in a yield of $60 \%$ and a m.p. of $111{ }^{\circ} \mathrm{C} .{ }^{1} \mathrm{H}$ NMR $\left(500 \mathrm{MHz}, \mathrm{CDCl}_{3}\right): \delta=2.33\left(\mathrm{~s}, 6 \mathrm{H}, \mathrm{CH}_{3}\right), 3.78\left(\mathrm{~s}, 3 \mathrm{H}, \mathrm{OCH}_{3}\right), 6.30$ $\left(\mathrm{d}, J=4.1 \mathrm{~Hz}, 1 \mathrm{H}\right.$. $\left.\mathrm{CH}_{\mathrm{th}}\right), 7.12(\mathrm{~s}, 8$ arom. H) $7.51(\mathrm{~d}, J=4.3 \mathrm{~Hz}$,

$\left.1 \mathrm{H}, \mathrm{CH}_{\mathrm{th}}\right)$.

Anal. Calcd for $\mathrm{C}_{20} \mathrm{H}_{19} \mathrm{NO}_{2} \mathrm{~S}$ (337.44): C 71.19, H 5.68, N 4.15; found C 71.37, H 5.60, N 4.28.

Methyl 5-(2,3-dihydro-indol-1-yl)-thiophene-2-carboxylate (9e), from

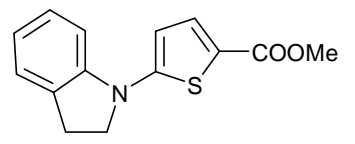
$\mathrm{N}$-acetyl-2,3-1H-dihydro-indol (11e) [19] via the corresponding 1chloro-1-( $N$-(2,3-dihydro- $1 H$-indolyl)-dimethylpropeniminium perchlorate (10f) in a yield of $56 \%$ and a m.p. of $95-97{ }^{\circ} \mathrm{C} .{ }^{1} \mathrm{H}$ NMR $\left(500 \mathrm{MHz}, \mathrm{CDCl}_{3}\right): \delta=3.27\left(\mathrm{t}, J=11.5 \mathrm{~Hz}, 2 \mathrm{H}, \mathrm{CH}_{2}\right), 3.87\left(\mathrm{~s}, 3 \mathrm{H}, \mathrm{OCH}_{3}\right), 4.05(\mathrm{t}, J=11.5 \mathrm{~Hz}$, $\left.2 \mathrm{H}, \mathrm{CH}_{2}\right), 6.33\left(\mathrm{~d}, J=6.0 \mathrm{~Hz}, 1 \mathrm{H}, \mathrm{CH}_{\mathrm{th}}\right), 6.91$ (t, $J=8.0 \mathrm{~Hz}, 1$ arom. H), 7.22 (t, $J=10.4 \mathrm{~Hz}, 2$ arom. H), 7.37 (d, $J=10.4 \mathrm{~Hz}, 1$ arom. H), $7.66\left(\mathrm{~d}, J=5.7 \mathrm{~Hz}, 1 \mathrm{H}, \mathrm{CH}_{\mathrm{th}}\right) ;{ }^{13} \mathrm{C} \mathrm{NMR}(125.76$ $\left.\mathrm{MHz} \mathrm{CDCl}_{3}\right): \delta=27.74,51.77,53.53,108.66,109.33,120.77,121.23,125.25,127.56,131.58$, 136.47, 144.45, 157.42, 164.37.

Anal. Calcd for $\mathrm{C}_{14} \mathrm{H}_{13} \mathrm{NO}_{2} \mathrm{~S}$ (259.32): C 64.84, H 5.05, N 5.40; found C 64.81, H 5.12, N 5.44.

\section{General procedure for preparation of $N, N$-disubstituted 2-amino-thiophene-5- $N$ ', $N$ '- dimethylmethiniminium perchlorates (12)}

To a mixture of a $N, N$-disubstituted 2-aminothiophene $(7,0.01 \mathrm{~mol})$ or a corresponding $N, N$ disubstituted 2-aminothiophene-5-carboxylic acid (8, $0.01 \mathrm{~mol})$ in DMF (25 mL) $\mathrm{POCl}_{3}(3.0 \mathrm{~g}$, $0.02 \mathrm{~mol}$ ) was added under stirring and cooling. The resulting mixture was heated at $60{ }^{\circ} \mathrm{C}$ for 20 min and mixed after cooling with methanol $(25 \mathrm{~mL})$ and perchloric acid $(70 \%, 3 \mathrm{~mL})$. The product precipitated after adding some diethyl ether was isolated by suction, dried in air, and recrystallised from acetic acid.

The following $\quad N, N$-disubstituted 2-amino-thiophene-5- $N^{\prime}, N^{\prime}$-dimethylmethiniminium perchlorates 12 were so obtained: 


\section{2-Diphenylamino-thiophene-5-dimethylmethiniminium perchlorate (12a),}

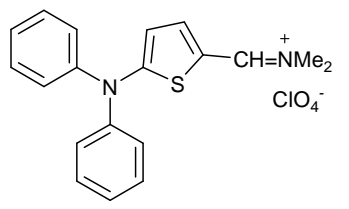

from 2-diphenylamino-thiophene (7a) in a yield of $80 \%$ or from 2diphenylamino-thiophene-5-carboxylic acid (8a) [16] a yield of $70 \%$ and a m.p. of $197-198{ }^{\circ} \mathrm{C} .{ }^{1} \mathrm{H}$ NMR $\left(500 \mathrm{MHz}, \mathrm{CF}_{3} \mathrm{COOH}\right): \delta=3.32$ (s, 3H, $\left.\mathrm{NCH}_{3}\right), 3.50\left(\mathrm{~s}, 3 \mathrm{H}, \mathrm{NCH}_{3}\right), 6.48\left(\mathrm{~d}, J=4.8 \mathrm{~Hz}, 1 \mathrm{H}, \mathrm{CH}_{\mathrm{th}}\right), 7.41$ -7.45 (m, 2 arom H), $7.52-7.56(\mathrm{~m}, 8$ arom. H), $7.94(\mathrm{~d}, J=4.8 \mathrm{~Hz}$, $\left.1 \mathrm{H}, \mathrm{CH}_{\mathrm{th}}\right), 8.70(\mathrm{~s}, 1 \mathrm{H}, \mathrm{CH}=\mathrm{N}) ;{ }^{13} \mathrm{C} \mathrm{NMR}\left(125.76 \mathrm{MHz}, \mathrm{CDCl}_{3}\right): \delta=43.37,115.31,117.51$, 119.83, 122.09, 129.82, 132.69, 134.18, 148.06, 163.81.

Anal. Calcd for $\mathrm{C}_{19} \mathrm{H}_{19} \mathrm{ClN}_{2} \mathrm{O}_{4} \mathrm{~S}$ (406.88): C 56.09, $\mathrm{H}$ 4.71, N 6.88; found C 55.97, H 4.75, N 6.69 .

5-[( $N$-(1-Naphtyl)- $N$-phenyl]amino-thiophene-2-dimethylmethiniminium perchlorate (12b),

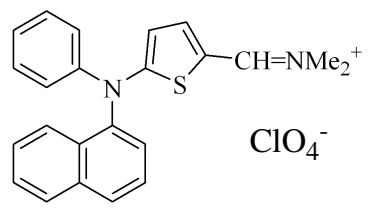

from 2-[(N-(1-naphtyl)- $N$-phenyl]amino-thiophene-5-carboxylic acid (8b) via the corresponding methyl 2-[(N-(1-naphtyl)-phenyl] aminothiophene-5-carboxylate (9b) in a yield of $62 \%$ and a m.p. of $197{ }^{\circ} \mathrm{C}$. ${ }^{1} \mathrm{H}$ NMR $\left(500 \mathrm{MHz}, \mathrm{CF}_{3} \mathrm{COOH}\right): \delta=3.17\left(\mathrm{~s}, 3 \mathrm{H}, \mathrm{NCH}_{3}\right), 3.35(\mathrm{~s}, 3 \mathrm{H}$, $\left.\mathrm{NCH}_{3}\right), 6.37\left(\mathrm{~s}, \mathrm{br}, 1 \mathrm{H}, \mathrm{CH}_{\mathrm{th}}\right), 7.23(\mathrm{~m}, 1$ arom. $\mathrm{H}), 7.31(\mathrm{t}, J=7.6$ $\mathrm{Hz}, 1$ arom. H), $7.40-7.46\left(\mathrm{~m}, 6\right.$ arom. H), $7.60\left(\mathrm{~d}, J=4.3 \mathrm{~Hz}, 1 \mathrm{H}, \mathrm{CH}_{\mathrm{th}}\right), 7.82(\mathrm{~s}, 1 \mathrm{H}, \mathrm{CH}=\mathrm{N})$, 7.84 - 7.88 (m, 4 arom. $\mathrm{H})$.

Anal. Calcd for $\mathrm{C}_{23} \mathrm{H}_{21} \mathrm{ClN}_{2} \mathrm{O}_{4} \mathrm{~S}$ (456.94): C 60.46, H 4.63, N 6.13; found $\mathrm{C} 60.73, \mathrm{H} 4.49, \mathrm{~N}$ 6.27 .

2-Phenothiazin-10-yl-thiophene-2-dimethylmethiniminium perchlorate (12e),

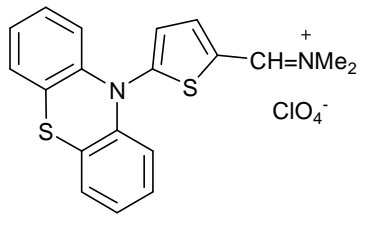

from 10-(2-thienyl)-10H-phenothiazine (7e) [16] in a yield of $80 \%$ and a m.p. of $198{ }^{\circ} \mathrm{C}$. ${ }^{1} \mathrm{H}$ NMR $\left(500 \mathrm{MHz}, \mathrm{CF}_{3} \mathrm{COOH}\right): \delta=2.92(\mathrm{~s}$, $\left.3 \mathrm{H}, \mathrm{NCH}_{3}\right), 3.33\left(\mathrm{~s}, 3 \mathrm{H}, \mathrm{NCH}_{3}\right), 5.68\left(\mathrm{~d}, J=11.0 \mathrm{~Hz}, 1 \mathrm{H}, \mathrm{CH}_{\mathrm{th}}\right), 7.22$ (t, 2 arom. H), 7.30 (t, 2 arom. H), 7.45 (dq, 4 arom. H), 7.66 (s, 1H, $\mathrm{CH}=\mathrm{N}), 8.10\left(\mathrm{~d}, J=4.0 \mathrm{~Hz}, 1 \mathrm{H}, \mathrm{CH}_{\mathrm{th}}\right)$.

Anal. Calcd for $\mathrm{C}_{19} \mathrm{H}_{17} \mathrm{ClN}_{2} \mathrm{O}_{4} \mathrm{~S}_{2}$ (436.93): C 52.23, H 3.92, N 6.41;

found C 52.44, H 3.96, N 6.53.

2-(3,4-Dihydro-2H-quinolin-1-yl)-thiophene-5-dimethyl-methiniminium perchlorate (12f), from 2-(3,4-dihydro-2H-quinolin-1-yl)-thiophene-5-carboxylic acid

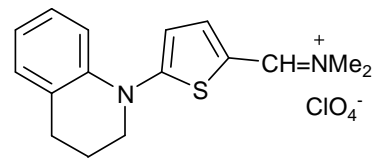
(8f), prepared from the corresponding methyl 2-(3,4-dihydro- $2 \mathrm{H}$ quinolin-1-yl)-thiophene-5-carboxylate ${ }^{[13 \mathrm{a}]}(\mathbf{9 g})$ in a yield of $85 \%$ and a m.p. of $204-211{ }^{\circ} \mathrm{C} .{ }^{1} \mathrm{H} \mathrm{NMR}\left(500 \mathrm{MHz}, \mathrm{CF}_{3} \mathrm{COOH}\right): \delta=2.01$ (q, $\left.J=6.3 \mathrm{~Hz}, 2 \mathrm{H}, \mathrm{CH}_{2}\right), 2.67\left(\mathrm{t}, J=6.2 \mathrm{~Hz}, 2 \mathrm{H}, \mathrm{CH}_{2}\right), 3.34(\mathrm{~s}, 3 \mathrm{H}$, $\left.\mathrm{NCH}_{3}\right) 3.38\left(\mathrm{~s}, 3 \mathrm{H}, \mathrm{NCH}_{3}\right), 3.76\left(\mathrm{t}, J=6.4 \mathrm{~Hz}, 2 \mathrm{H}, \mathrm{CH}_{2}\right), 6.80\left(\mathrm{~d}, J=5.0 \mathrm{~Hz}, 1 \mathrm{H}, \mathrm{CH}_{\mathrm{th}}\right), 7.08$ (m, 1 arom. H), 7.16 (m, 2 arom. H), 7.46, d, $J=8.0 \mathrm{~Hz}, 1$ arom. H), 7.66, d, $J=5.1 \mathrm{HZ}, 1 \mathrm{H}$, $\left.\mathrm{CH}_{\mathrm{th}}\right), 7.88(\mathrm{~s}, 1 \mathrm{H}, \mathrm{CH}=\mathrm{N})$.

Anal. Calcd for $\mathrm{C}_{16} \mathrm{H}_{19} \mathrm{ClN}_{2} \mathrm{O}_{4} \mathrm{~S}$ (370.85) C 51.82, H 5.16, N 7.55; found $\mathrm{C} 51.65, \mathrm{H} \mathrm{5.22,} \mathrm{N}$ 7.50. 
General procedure for preparation of $N, N$-disubstituted methyl 2-amino-5-formylthiophene-5-carboxylates (13) or 2-amino-3-formyl-thiophene-5-carboxylates (17)

To a solution of a $N, N$-disubstituted 2-aminothiophene 7, a $N, N$-disubstituted 2-aminothiophene5-carboxylic acid 8, or a $N, N$-disubstituted methyl 2-aminothiophene-5-carboxylate 9 (0.01 mol, each) in DMF $(25 \mathrm{~mL}) \mathrm{POCl}_{3}(3.0 \mathrm{~g}, 0.02 \mathrm{~mol})$ was added under stirring and cooling. The resulting mixture was left standing at room temperature or heated at $60{ }^{\circ} \mathrm{C}$ for $20 \mathrm{~min}$ and mixed after cooling with methanol $(25 \mathrm{~mL})$ and water $(50 \mathrm{~mL})$. After addition of aqueous sodium hydroxide until weak basic reaction the products precipitated after some standing at room temperature and were isolated by suction, dried in air and recrystallised from cyclohexane or toluene.

The following $N, N$-disubstituted 2-amino-thiophene-5-carbaldehydes $\mathbf{1 3}$ and 2-amino-thiophene3-carbaldehydes 17 were so obtained:

2-Diphenylamino-thiophene-5-carbaldehyde (13a),

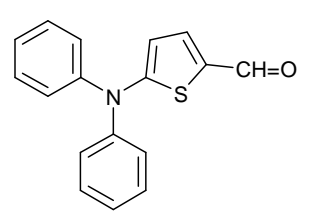

from 2-diphenylaminothiophene (7a) [16] in a yield of $85 \%$ or from 2diphenylamino-thiophene-5-carboxylic acid (8a) [16] in a yield of $76 \%$ with a m.p. $92{ }^{\circ} \mathrm{C} .{ }^{1} \mathrm{H}$ NMR $\left(500 \mathrm{MHz}, \mathrm{CDCl}_{3}\right): \delta=6.38(\mathrm{~d}, J=4.4 \mathrm{~Hz}$, $\left.1 \mathrm{H}, \mathrm{CH}_{\mathrm{th}}\right), 7.22$ (t, J=7.3 Hz, 2 arom. H), $7.27-7.28$ (m. 4 arom. H), $7.34-7.37$ (m, 4 arom. H), $7.46\left(\mathrm{~d}, J=4.4 \mathrm{~Hz}, 1 \mathrm{H}, \mathrm{CH}_{\mathrm{th}}\right), 9.56(\mathrm{~s}, 1 \mathrm{H}$, $\mathrm{CH}=\mathrm{O}) ;{ }^{13} \mathrm{C} \mathrm{NMR}\left(125.76 \mathrm{MHz}, \mathrm{CF}_{3} \mathrm{COOH}\right): \delta=112.40,125.48,126.36,129.62,129.84$, 139.22, 145.75, 165.25. 180.73 .

Anal. Calcd for $\mathrm{C}_{17} \mathrm{H}_{13} \mathrm{NOS}$ (279.36): C 73.09, H 4.69, N 5.01; found C 73.16, H 4.60, N 4.93.

2-[( $N$-(1-Naphtyl)- $N$-phenyl] amino-thiophene-5-carbaldehyde (13b),

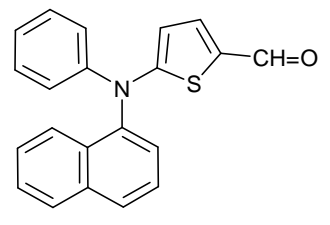

from 2-[( $N$-(1-naphtyl)- $N$-phenyl]amino-thiophene-5-carboxylic acid (8b) via the corresponding methyl 2-[( $N$-(1-naphtyl)- $N$-phenyl $]$ aminothiophene-5-carboxylate $(\mathbf{9 b})$ in a yield of $62 \%$ as pale yellow oil. ${ }^{1} \mathrm{H}$ $\operatorname{NMR}\left(500 \mathrm{MHz}, \mathrm{CDCl}_{3}\right): \delta=6.28\left(\mathrm{~s}, \mathrm{br}, 1 \mathrm{H}, \mathrm{CH}_{\mathrm{th}}\right), 7.17-7.18(\mathrm{~m}, 1$ arom. H), $7.32-7.38$ (m, 4 arom. H), $7.46-7.54$ (m, 5 arom H), 7.90 - 7.94 (m, 2 arom. H., 1 hetarom H), 9.39 (s, br, 1H, CHO).

Anal. Calcd for $\mathrm{C}_{21} \mathrm{H}_{15} \mathrm{NOS}$ (329.61): C 76.57, H 4.59, H 4.25, O 4.86, S 9.73; found C 76.76, H 4.64, N 4.51.

Methyl 2-dimethylamino-3-formyl-thiophene-5-carboxylate (17a),

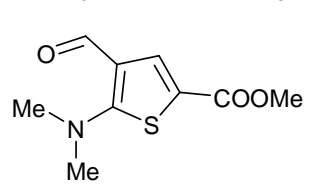
from methyl 2-dimethylamino-thiophene-5-carboxylate ${ }^{13 \mathrm{a}}(\mathbf{9 h})$ in a yield of $60 \%$ and a m.p. of $110{ }^{\circ} \mathrm{C}$. ${ }^{1} \mathrm{H}$ NMR $\left(500 \mathrm{MHz}, \mathrm{CDCl}_{3}\right): \delta=3.42$ (s, $\left.3 \mathrm{H}, \mathrm{OCH}_{3}\right) 3.51\left(\mathrm{~s}, 3 \mathrm{H}, \mathrm{NCH}_{3}\right), 3.90\left(\mathrm{~s}, 3 \mathrm{H}, \mathrm{NCH}_{3}\right), 7.97\left(\mathrm{~s}, 1 \mathrm{H}, \mathrm{CH}_{\mathrm{th}}\right)$, $8.87(\mathrm{~s}, 1 \mathrm{H}, \mathrm{CHO})$.

Anal. Calcd for $\mathrm{C}_{9} \mathrm{H}_{11} \mathrm{NO}_{3} \mathrm{~S}$ (213.05): C 50.69, H 5.20, N 6.57; found C 50.73, H 5.22, N 6.49.

\section{Methyl 2-(4-morpholinyl)-3-formyl-thiophene-3-carboxylate (17b),}

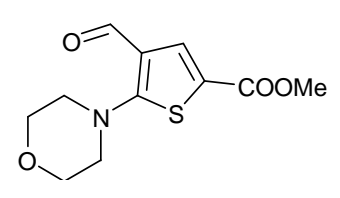

from methyl 2-(4-morpholinyl)-thiophene-5-carboxylate ${ }^{13 \mathrm{a}}(\mathbf{9 j})$ in a yield of $72 \%$ and a m.p. of $118{ }^{\circ} \mathrm{C} .{ }^{1} \mathrm{H}$ NMR $\left(500 \mathrm{MHz}, \mathrm{CDCl}_{3}\right): \delta=3.68(\mathrm{t}$, 
$\left.J=4.8 \mathrm{~Hz}, 4 \mathrm{H}, \mathrm{CH}_{2}\right), 3.90\left(\mathrm{~s}, 3 \mathrm{H}, \mathrm{OCH}_{3}\right), 4.08\left(\mathrm{t}, J=4.8 \mathrm{~Hz}, 4 \mathrm{H}, \mathrm{CH}_{2}\right), 7.99\left(\mathrm{~s}, 1 \mathrm{H}, \mathrm{CH}_{\mathrm{th}}\right), 9.32$ $(\mathrm{s}, 1 \mathrm{H}, \mathrm{CHO}) ;{ }^{13} \mathrm{C} \mathrm{NMR}\left(125.76 \mathrm{MHz}, \mathrm{CDCl}_{3}\right): \delta=45.94,51.99,115.30,129.82,137.41$, $162.35,169.72,182.09$.

Anal. Calcd for $\mathrm{C}_{11} \mathrm{H}_{13} \mathrm{NO}_{4} \mathrm{~S}$ (255.29): C 51,75, H 5.13, N 5.49; found C 51.63, H 5.22, N 5.58.

\section{General procedure for preparation of $N$-substituted 2-methoxycarbonyl-thieno[2,3- b]quinolinium perchlorates (18)}

To a solution of a $N$-substituted methyl $N$-aryl-2-aminothiophene-5-carboxylate $(\mathbf{9}, 0.01 \mathrm{~mol})$ in DMF $(25 \mathrm{~mL}) \mathrm{POCl}_{3}(3.0 \mathrm{~g}, 0.02 \mathrm{~mol})$ was added under stirring and cooling. The resulting mixture was heated at $60{ }^{\circ} \mathrm{C}$ for $20 \mathrm{~min}$ and mixed after cooling with methanol $(25 \mathrm{~mL})$ and perchloric acid $(70 \%, 3 \mathrm{~mL})$. The product precipitated after addition of some diethyl ether was isolated by suction, dried at air, and recrystallised from acetic acid.

The following $N$-substituted 2-methoxycarbonyl-thieno[2,3-b]quinolinium perchlorates (18) were so obtained:

\section{2-Methoxycarbonyl-9-phenyl-thieno[2,3-b]quinolinium perchlorate (18a),}

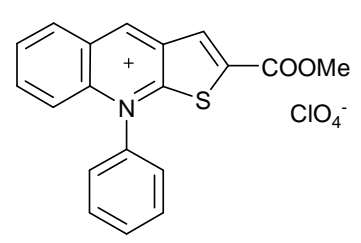

from methyl 2-(N,N-diphenylamino)-thiophene-5-carboxylate ${ }^{13 a}(\mathbf{9 a})$ in a yield of $90 \%$ and a m.p. of $293-295{ }^{\circ} \mathrm{C}$. ${ }^{1} \mathrm{H}$ NMR $(500 \mathrm{MHz}$, DMSO-D6): $\delta=3.96\left(\mathrm{~s}, 3 \mathrm{H}, \mathrm{CH}_{3} \mathrm{O}\right), 7.63(\mathrm{~d}, J=12.0 \mathrm{~Hz}, 1$ arom $\mathrm{H})$, $7.95-7.98$ ( m, 5 arom. H), 8.10 (m, 1 arom. H), 8.27 (m, 1 arom. H), $8.76\left(\mathrm{~s}, 1 \mathrm{H}, \mathrm{CH}_{\mathrm{th}}\right), 8,78(\mathrm{~s}, 1$ arom $\mathrm{H}), 10.05$ (s, 1 arom. $\left.\mathrm{H}\right)$.

MS (ESI, $70 \mathrm{eV}): \mathrm{m} / \mathrm{z}=320.0$; calcd for $\mathrm{C}_{19} \mathrm{H}_{14} \mathrm{NO}_{2} \mathrm{~S}^{+}=320.38 ;{ }^{13} \mathrm{C} \mathrm{NMR}(125.76 \mathrm{MHz}$, $\left.\mathrm{CF}_{3} \mathrm{COOH}\right): \delta=53.13,112.01,124.66,126.44,128.48,129.67,130.71,131.09,132.36,132.38$, $134.34,137.27,137.37,140.26,144.65,161.73,162.53$.

Anal. Calcd for $\mathrm{C}_{19} \mathrm{H}_{14} \mathrm{ClNO}_{6} \mathrm{~S}$ (419.84): C 54.36, H 3.36, N 3.34; found C 54.55, H 3.28, N 3.46.

\section{9-Methoxycarbonyl-11-phenyl-benzo[h]thieno[2,3-b]quinolinium perchlorate $(18 \mathrm{~b})$,}

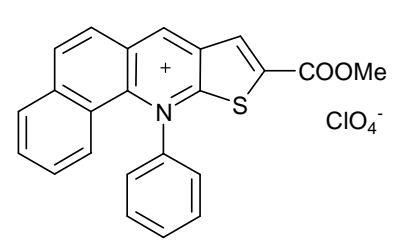

from methyl 2-[N-phenyl- $N$-(1-naphthyl)]-amino-thiophene-5carboxylate (9b) in a yield of $88 \%$ and a m.p. of $300-301{ }^{\circ} \mathrm{C} .{ }^{1} \mathrm{H}$ NMR (500 MHz, DMSO-D6): $\delta=3.90\left(\mathrm{~s}, 3 \mathrm{H}, \mathrm{CH}_{3} \mathrm{O}\right), 7.18(\mathrm{~d}, J=$ $10.8 \mathrm{~Hz}, 1$ arom. H), $7.47-7.52$ (m, 2 arom. H), 7.76 (t, $J=10.0$ $\mathrm{Hz}, 1$ arom. H), 8.01 (t, $J=10.4 \mathrm{~Hz}, 1$ arom. $\mathrm{H}), 8.10-8.23(\mathrm{~m}, 4$ arom. H), 8.33 (d, $J=10.8 \mathrm{~Hz}, 1$ arom. H), $8.56(\mathrm{~d}, J=10.8 \mathrm{~Hz}, 1$

arom $\mathrm{H}), 8.81$ (s, 1 arom. H), 8.86 (dd, $J=10.8$ and $2.2 \mathrm{~Hz}, 2$ arom. H), 10.18 (s, 1 arom. H); ${ }^{13} \mathrm{C}$ NMR (125.76 MHz, $\left.\mathrm{CF}_{3} \mathrm{COOH}\right): \delta=53.12,117.19,119.20,124.89,124.30,125.49,126.42$, $127.56,128.58,128.69$, 128.86, 129.67, 130.94, 132.56, 133.05, 133.29, 134.46, 134.66, 137.59, $140.57,145.11,162.44,162.78$.

MS (ESI, $70 \mathrm{eV}$ ): $\mathrm{m} / \mathrm{z}=370.0$; calcd for $\mathrm{C}_{23} \mathrm{H}_{16} \mathrm{NO}_{2} \mathrm{~S}^{+}=370.44$.

Anal. Calcd for $\mathrm{C}_{23} \mathrm{H}_{16} \mathrm{ClNO}_{6} \mathrm{~S}$ (469.89): C 58.79, H 3.43, N 2.98; found C 58.55, H 3.13, N 3.10 . 
9-Methoxycarbonyl-7-phenyl-benzo[f]thieno[2,3-b]quinolinium perchlorate (18c),

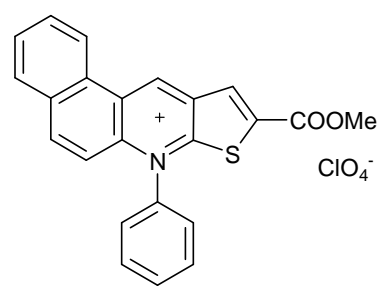

from methyl 2-[N-phenyl- $N$-(2-naphthyl]-amino-thiophene-5carboxylate (9c) in a yield of $92 \%$ and a m.p. of $330-332{ }^{\circ} \mathrm{C}$. ${ }^{1} \mathrm{H}$ NMR (500 MHz, DMSO-D6): $\delta=3.98$ (s, 3H, $\left.\mathrm{CH}_{3} \mathrm{O}\right), 7.54$ $(\mathrm{d}, J=12.4 \mathrm{~Hz}, 1$ arom. H), $7.89-8.00(\mathrm{~m}, 5$ arom $\mathrm{H}), 8.03(\mathrm{t}, 1$ arom $\mathrm{H}), 8.15$ (t, 1 arom. $\mathrm{H}), 8.35(\mathrm{~d}, J=10.4 \mathrm{~Hz}, 1$ arom. $\mathrm{H})$, 8.65 (d, $J=12.8 \mathrm{~Hz}, 1$ arom. H), 8.69 (s, 1 arom. H), 9.20 (d, $J=$

$11.2 \mathrm{~Hz}, 1$ arom. $\mathrm{H}), 10.87$ (s, 1 arom $\left.\mathrm{H}) ;{ }^{13} \mathrm{C} \mathrm{NMR} \mathrm{(125.76} \mathrm{MHz,} \mathrm{CF}_{3} \mathrm{COOH}\right): \delta=53.13$, $113.89,122.00,124.74,125.42,127.68,129.26,129.44,130.00,130.08,130.62,131.14,132.13$, $132.39,134.36,137.87,140.82,141.93,158.40,162.83$.

MS (ESI, $70 \mathrm{eV}$ ): $\mathrm{m} / \mathrm{z}=370.0$; calcd for $\mathrm{C}_{23} \mathrm{H}_{16} \mathrm{NO}_{2} \mathrm{~S}^{+}=370.44$.

Anal. Calcd for $\mathrm{C}_{23} \mathrm{H}_{16} \mathrm{ClNO}_{6} \mathrm{~S}$ (469.89): C 58.79, H 3.43, N 2.98; found C 59.02, H 3.25, N 3.07 .

\section{2-Methoxycarbonyl-6-methyl-9-(4-tolyl)-thieno[2,3-b]quinolinium perchlorate (18d),}

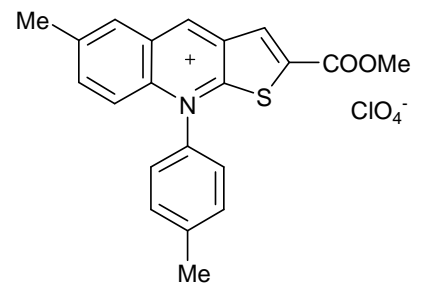

from methyl 2-(N,N-(4-tolyl)]-aminothiophene-5-carboxylate (9d) in a yield of $60 \%$ and a m.p. of $313-315^{\circ} \mathrm{C} .{ }^{1} \mathrm{H}$ NMR $(500 \mathrm{MHz}$, DMSO-D6): $\delta=2.52\left(\mathrm{~s}, 3 \mathrm{H}, \mathrm{CH}_{3}\right), 2.65\left(\mathrm{~s}, 3 \mathrm{H}, \mathrm{CH}_{3}\right), 3.95(\mathrm{~s}, 3 \mathrm{H}$, $\left.\mathrm{CH}_{3} \mathrm{O}\right), 7.14(\mathrm{~d}, J=11.6 \mathrm{~Hz}, 1$ arom. $\mathrm{H}) 7.72-7.80(\mathrm{~m}, 4$ arom $\mathrm{H})$, $7.56(\mathrm{dd}, J=12.0$ and $2.0 \mathrm{~Hz}, 1$ arom. H), 8.51 (s, 1 arom. H), 8.71 (s, 1 arom. H), 9.91 (s, 1 arom. H).

MS (ESI, $70 \mathrm{eV}$ ): $\mathrm{m} / \mathrm{z}=348.1$; calcd for $\mathrm{C}_{21} \mathrm{H}_{18} \mathrm{NO}_{2} \mathrm{~S}^{+}=348.44$.

Anal. Calcd for $\mathrm{C}_{21} \mathrm{H}_{18} \mathrm{ClNO}_{6} \mathrm{~S}$ (447.89): C 56.31, H 4.05, N 3.13 found C 56.18, H 4.25, N 3.00.

\section{1,2-Dihydro-7-methoxycarbonyl-cyclopenta[1,2-i,j]quinolinium perchlorate (18e),}

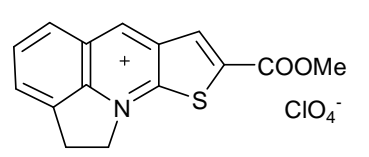

from methyl 2-(2,3-dihydro-indol-1-yl)-thiophene-5-carboxylate (9e) in a yield of $60 \%$ and a m.p. of $250-251{ }^{\circ} \mathrm{C} .{ }^{1} \mathrm{H} \mathrm{NMR}(500 \mathrm{MHz}$, DMSO-D6): $\delta=3.97\left(\mathrm{t}, J=9.2 \mathrm{~Hz}, 2 \mathrm{H}, \mathrm{CH}_{2}\right), 4.03\left(\mathrm{~s}, \mathrm{H}, \mathrm{OCH}_{3}\right), 5.36$ $\left(\mathrm{t}, J=9.6 \mathrm{~Hz}, 2 \mathrm{H}, \mathrm{CH}_{2}\right), 7.99(\mathrm{t}, J=10.6 \mathrm{~Hz}, 1$ arom $\mathrm{H}), 8.15(\mathrm{~d}, J=$ $9.6 \mathrm{~Hz}, 1$ arom. H), 8.37 (d, $J=11.2 \mathrm{~Hz}, 1$ arom. H), 8.71 (s, 1 arom. H), 9.74 (s, 1 arom. H).

MS (ESI, $70 \mathrm{eV}): \mathrm{m} / \mathrm{z}=269.9$; calcd for $\mathrm{C}_{15} \mathrm{H}_{12} \mathrm{NO}_{2} \mathrm{~S}^{+}=270.33 ;{ }^{13} \mathrm{C} \mathrm{NMR}(125.76 \mathrm{MHz}$, $\left.\mathrm{CF}_{3} \mathrm{COOH}\right): \delta=$.

Anal. Calcd for $\mathrm{C}_{15} \mathrm{H}_{12} \mathrm{ClNO}_{6} \mathrm{~S}$ (369.8): C 48.72, H 3.27, N 3.79; found C 48.48, H 3.33, N 3.57.

2,3-Dihydro-1H-7-methoxycarbonyl-benzo[1,2-i,j]quinolinium perchlorate (18f),

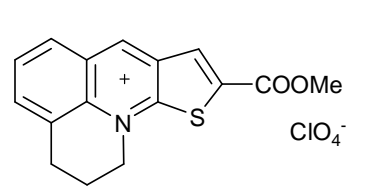

from methyl 2-(3,4-dihydro-2H-quinolin-1-yl)-thiophene-5carboxylate $^{13 \mathrm{a}}(\mathbf{9 f})$ in a yield of $74 \%$ and a m.p. of $278-279{ }^{\circ} \mathrm{C} .{ }^{1} \mathrm{H}$ NMR (500 MHz, $\left.\mathrm{CF}_{3} \mathrm{COOH}\right): \delta=3.03\left(\mathrm{q}, 2 \mathrm{H}, \mathrm{CH}_{2}\right), 3.82(\mathrm{t}, J=8.0$ $\left.\mathrm{Hz}, 2 \mathrm{H}, \mathrm{CH}_{2}\right), 4.53\left(\mathrm{~s}, 3 \mathrm{H}, \mathrm{OCH}_{3}\right), 5.35\left(\mathrm{t}, J=8.0 \mathrm{~Hz}, 2 \mathrm{H}, \mathrm{CH}_{2}\right), 8.29$ (t, $J=10.8 \mathrm{~Hz}, 1$ arom. H), $8.45(\mathrm{~d}, J=9.6 \mathrm{~Hz}, 1$ arom. H), $8.65(\mathrm{t}, J=10.8 \mathrm{~Hz}, 1$ arom. H), 8.92 (s, 1 arom. H), 9.82 (s, 1 arom. H); ${ }^{13} \mathrm{C}$ NMR (125.76 MHz, $\left.\mathrm{CF}_{3} \mathrm{COOH}\right): \delta=19.14,24.86,53.24$, 
$54.65,126.80,127.57,128.08,128.95,130.05,132.14,133.21,134.73,135.54,143.33,157.96$, 162.63.

MS (ESI, $70 \mathrm{eV}$ ): $\mathrm{m} / \mathrm{z}=284.0$; cald for $\mathrm{C}_{16} \mathrm{H}_{14} \mathrm{NO}_{2} \mathrm{~S}^{+}=284.35$.

Anal. Calcd for $\mathrm{C}_{16} \mathrm{H}_{14} \mathrm{ClNO}_{6} \mathrm{~S}$ (383.80): C 50.07, H 3.68, N 3.65; found C 49.88, H 3.58, N 3.46 .

2-Methoxycarbonyl-9-methyl-thieno[2,3-b]quinolinium perchlorate $(18 \mathrm{~h})$,

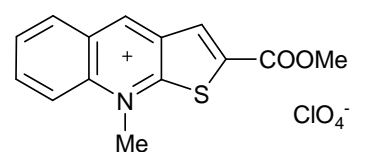

from methyl $N$-methyl- $N$-phenyl-2-amino-thiophene-5-carboxylate ${ }^{13 \mathrm{a}}$ (9h) in a yield of $70 \%$ and a m.p. of $265-268{ }^{\circ} \mathrm{C} .{ }^{1} \mathrm{H}$ NMR $(500$ MHz, DMSO-D6): $\delta=4.53\left(\mathrm{~s}, 3 \mathrm{H}, \mathrm{NCH}_{3}\right), 5.17\left(\mathrm{~s}, 3 \mathrm{H}, \mathrm{OCH}_{3}\right), 8.42(\mathrm{t}$, $J=9.6 \mathrm{~Hz}, 1$ arom. H), $8.47-8.85(\mathrm{~m}, 3$ arom $\mathrm{H}), 8.91$ (s, 1 arom. $\mathrm{H})$, 9.90 (s, 1 arom $\mathrm{H}) ;{ }^{13} \mathrm{C}$ NMR (125.76 MHz, $\left.\mathrm{CF}_{3} \mathrm{COOH}\right): \delta=41.50,53.25,115.50,126.45$, $128.40,130.11,131.30,132.50,133.12,137.52,130.28,143.84,162.44$.

MS (ESI, $70 \mathrm{eV}$ ): $\mathrm{m} / \mathrm{z}=257.9$; Calcd for $\mathrm{C}_{14} \mathrm{H}_{12} \mathrm{NO}_{2} \mathrm{~S}^{+}=258.32$.

Anal. Calcd for $\mathrm{C}_{14} \mathrm{H}_{12} \mathrm{ClNO}_{6} \mathrm{~S}$ (357.77): C 47.00, H 3.38, N 3.92; found $\mathrm{C} 47.22, \mathrm{H} \mathrm{3.30,} \mathrm{Cl}$ 10.12, N 4.02, S 9.16.

\section{2-Methoxycarbonyl-4-[5-( $N$-morpholinyl)-2-thienyl]-9-phenyl-thieno[2,3-b]quinolinium perchlorate (19)}

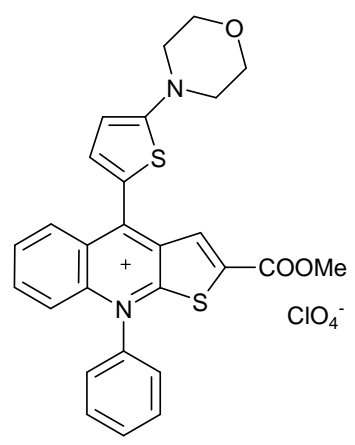

A mixture of 2-methoxycarbonyl-9-phenyl-thieno[2,3-b]quinolinium perchlorate $(\mathbf{1 8 b}, 0.01 \mathrm{~mol})$ and 2-morpholinothiophene-5-carboxylic acid $8 \mathbf{j}(0.02 \mathrm{~mol})$ in acetic anhydride $(20 \mathrm{~mL})$ was refluxed for $4 \mathrm{~h}$. After cooling the reaction mixture and adding some diethyl ether the product formed crystals and it was isolated by suction in a yield of 42 $\%$ and a m.p. of $234-237{ }^{\circ} \mathrm{C}$. ${ }^{1} \mathrm{H}$ NMR (500 MHz, DMSO-D6): 3.68 $\left(\mathrm{t}, J=4.8 \mathrm{~Hz}, 4 \mathrm{H}, \mathrm{CH}_{2}\right), 3.84\left(\mathrm{t}, J=4.8 \mathrm{~Hz}, 4 \mathrm{H}, \mathrm{CH}_{2}\right), 3.88(\mathrm{~s}, 3 \mathrm{H}$, $\left.\mathrm{OCH}_{3}\right), 7.02\left(\mathrm{~d}, J=4.9 \mathrm{~Hz}, 1 \mathrm{H}, \mathrm{CH}_{\mathrm{th}}\right), 7.27(\mathrm{~d}, J=8.7 \mathrm{~Hz}, 1$ arom. H), $7.84-7.90$ (m, 5 arom. H), 8.00 (dt, 2 arom. H), 8.11 (d, $J=4.8$ $\left.\mathrm{Hz}, 1 \mathrm{H} \mathrm{CH}_{\mathrm{th}}\right), 8.57$ (s, $\left.1 \mathrm{H}, \mathrm{CH}_{\mathrm{th}}\right), 8.67$ (d, $J=8.6 \mathrm{~Hz}, 1$ arom. $\left.\mathrm{H}\right)$.

Anal. Calcd for $\mathrm{C}_{27} \mathrm{H}_{23} \mathrm{ClN}_{2} \mathrm{O}_{7} \mathrm{~S}_{2}$ (587.06): $\mathrm{C} 55.24, \mathrm{H} 3.95, \mathrm{~N} 4.77$; found $\mathrm{C}$ 55.67, $\mathrm{H} \mathrm{4.12,} \mathrm{N}$ 4.69.

\section{General procedure for preparation of methyl 4,9-dihydro-thieno[2,3-b]quinoline- carboxylates (20)}

To a mixture of a thieno[2,3-b]quinolinium salt $18(0.01 \mathrm{~mol})$ in methanol $(25 \mathrm{~mL})$ or DMF $(25$ $\mathrm{mL})$ sodium borotetrahydride $(0.8 \mathrm{~g}, 0.02 \mathrm{~mol})$ was added under stirring at room temperature. After finishing the gas evolution the reaction mixture was diluted with water $(100 \mathrm{~mL})$ and the precipitate formed isolated by suction.

The following 4,9-dihydro-thieno[2,3-b]quinoline-carboxylates 20 were so prepared.

\section{2-Methoxycarbonyl-9-phenyl-4,9-dihydrothieno[2,3-b]quinoline (20a),}

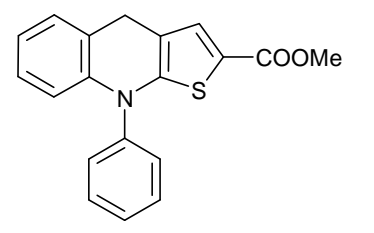

from 2-methoxycarbonyl-9-phenyl-thieno[2,3-b]quinolinium perchlorate 18a in a yield of $68 \%$ and a m.p. of $233{ }^{\circ} \mathrm{C} .{ }^{1} \mathrm{H}$ NMR $(500 \mathrm{MHz}$, 
$\left.\mathrm{CDCl}_{3}\right): \delta=3.75\left(\mathrm{~s}, 3 \mathrm{H}, \mathrm{OCH}_{3}\right), 4.23\left(\mathrm{~s}, 2 \mathrm{H}, \mathrm{CH}_{2}\right), 7.50(\mathrm{dd}, J=8.3,1.0 \mathrm{~Hz}, 1$ arom. H), 6.26( d, $J=8.3 \mathrm{~Hz}, 1$ arom. H), 6.90 (t, 1 arom. H), 6.97 (t, 1 arom. H), $7.38-7.39$ (m, 2 arom. H) $7.46\left(\mathrm{~s}, 1 \mathrm{H}, \mathrm{CH}_{\mathrm{th}}\right), 7.48-7.51(\mathrm{~m}, 1$ arom. $\mathrm{H}), 7.56-7.59(\mathrm{~m}, 2$ arom. $\mathrm{H}) ;{ }^{13} \mathrm{C}$ NMR $(125.76$ $\mathrm{MHz}, \mathrm{CCl}): \delta=27.40,50.06,112.10,118.73,122.05,125.95,126.30,125.46,126.30,127.21$, 127.42, 128.80, 128.89, 132.62, 151.25, 160.45.

Anal. Calcd for $\mathrm{C}_{19} \mathrm{H}_{15} \mathrm{NO}_{2} \mathrm{~S}$ (321.39): C 71.00, $\mathrm{H} 4.70, \mathrm{~N} 4.36$; found $\mathrm{C} 71.18, \mathrm{H} 4.72, \mathrm{~N} 4.61$.

9-Methoxycarbonyl-11-phenyl-4,9-dihydro-benzo[h]thieno [2,3-b]quinoline (20b),

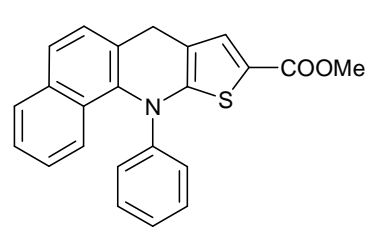

from 9-methoxycarbonyl-11-phenyl-benzo[h]thieno[2,3b]quinolinium perchlorate $\mathbf{1 8 b}$ in a yield of $75 \%$ and a m.p. of $186-$ $192{ }^{\circ} \mathrm{C}$. ${ }^{1} \mathrm{H}$ NMR $\left(500 \mathrm{MHz}, \mathrm{CDCl}_{3}\right): \delta=3.69\left(\mathrm{~s}, 3 \mathrm{H}, \mathrm{OCH}_{3}\right), 4.36(\mathrm{~s}$, $\left.2 \mathrm{H}, \mathrm{CH}_{2}\right), 6.07(\mathrm{~d}, J=9.1 \mathrm{~Hz}, 1$ arom. $\mathrm{H}), 6.86-6.91$ (m, 2 arom. $\left.\mathrm{H}\right)$, $7.18(\mathrm{~d}, J=7.9 \mathrm{~Hz}, 1$ arom. H), $7.42-7.43$ (m, 1 arom. H), $7.50-$ 7.54 (m, 2 arom. H), $7.58-7.64$ (m, 2 arom. H), 7.77 (d, $J=8.5 \mathrm{~Hz}, 1$ arom. H), 8.01 (d, $J=8.0$ $\mathrm{Hz}, 1$ arom. H), 8.16 (d, $J=8.3 \mathrm{~Hz}, 1$ arom. H); ${ }^{13} \mathrm{C}$ NMR (125.76 MHz, CCl4): $\delta=27.42$, 50.08, 112.10, 113.52, 117.33. 118.74, 121.14, 122.30, 123.39, 126.59, 127.78, 128.81, 128.91, $129.77,132.62,134.44,136.78,139.78,151.26,160.45$.

Anal. Calcd for $\mathrm{C}_{23} \mathrm{H}_{17} \mathrm{NO}_{2} \mathrm{~S}$ (371.45): C 74.37, H 4.61, N 3.77; found C 74.74, H 4.68, N 3.72.

9-Methoxycarbonyl-7-phenyl-4,9-dihydrobenzo[f]thieno[2,3-b]quinoline (20c),

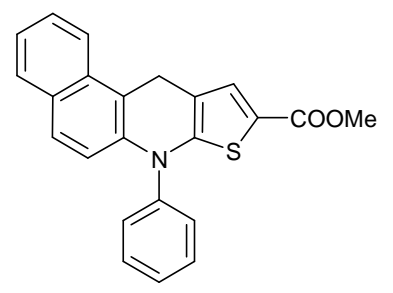

from 9-methoxycarbonyl-7-phenyl-benzo[f]thieno[2,3-b]quinolinium perchlorate 18c in a yield of $84 \%$ and a m.p. of $265-272{ }^{\circ} \mathrm{C} .{ }^{1} \mathrm{H}$ $\operatorname{NMR}\left(500 \mathrm{MHz}, \mathrm{CDCl}_{3}\right): \delta=3.78\left(\mathrm{~s}, 3 \mathrm{H}, \mathrm{OCH}_{3}\right), 4.57\left(\mathrm{~s}, 2 \mathrm{H}, \mathrm{CH}_{2}\right)$, $6.62(\mathrm{~d}, J=9.1 \mathrm{~Hz}, 1$ arom. H), $7.23-7.43$ (m 3 arom. H), $7.50-$ 7.54 (m, 2 arom. H), $7.56-7.63$ (m, 4 arom. H), 7.74 (d, $J=8.0 \mathrm{~Hz}, 1$ arom. H), 7.812 (d, $J=8.5 \mathrm{~Hz}, 1$ arom. $\mathrm{H}) ;{ }^{13} \mathrm{C}$ NMR $(125.76 \mathrm{MHz}$, $\left.\mathrm{CD}_{2} \mathrm{Cl}_{2}\right): \delta=25.91,51.92,112.05,114.56,115.24,116.24,118.17$,

$122.55,124.12,124.53,127.93,128.48,129.94,130.04,131.25,137.51,141.16,142.04,153.20$, 163.15 .

Anal. Calcd for $\mathrm{C}_{23} \mathrm{H}_{17} \mathrm{NO}_{2} \mathrm{~S}$ (371.45): C 74.37, $\mathrm{H} 4$ 4.61, N 3.77; found C 74.31, H 4.28, N 3.58.

9-Methoxycarbonyl-2,3-dihydro-1H,7H-pyrido[3,2,1-ij]thieno[2,3-b]quinoline (20f),

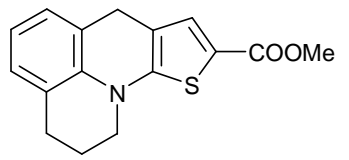

from 2,3-dihydro- $1 H$-7-methoxycarbonyl-benzo[1,2-i,j]quinolinium perchlorate 18f in a yield of $50 \%$ and a m.p. of $138-141{ }^{\circ} \mathrm{C} .{ }^{1} \mathrm{H}$ NMR $\left(500 \mathrm{MHz}, \mathrm{CDCl}_{3}\right): \delta=2.12\left(\mathrm{q}, 2 \mathrm{H}, \mathrm{CH}_{2}\right), 2.81\left(\mathrm{t}, J=6.2 \mathrm{~Hz}, 2 \mathrm{H}, \mathrm{CH}_{2}\right)$, $3.58\left(\mathrm{t}, J=5.8 \mathrm{~Hz}, 2 \mathrm{H}, \mathrm{CH}_{2}\right), 3.82\left(\mathrm{~s}, 3 \mathrm{H}, \mathrm{OCH}_{3}\right), 4.05\left(\mathrm{~s}, 2 \mathrm{H}, \mathrm{CH}_{2}\right)$, $6.81\left(\mathrm{t}, J=7.4 \mathrm{~Hz}, 2\right.$ arom. H), 6.90 (t, $J=6.4 \mathrm{~Hz}, 1$ arom H), $7.45\left(\mathrm{~s}, 1 \mathrm{H}, \mathrm{CH}_{\mathrm{th}}\right)$.

Anal. Calcd for $\mathrm{C}_{16} \mathrm{H}_{15} \mathrm{NO}_{2} \mathrm{~S}$ (285.36): C 67.34, H 5.30, N 4.91; found C 67.48, H 5.12, N 5.08.

\section{2-Methoxycarbonyl-9-methyl-4,9-dihydro-thieno[2,3-b]quinoline (20h),}

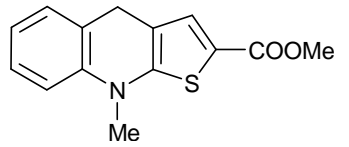

from 2-methoxycarbonyl-9-methyl-thieno[2,3-b]quinolinium perchlorate $\mathbf{1 8 h}$ in a yield of $70 \%$ and a m.p. of $115-120{ }^{\circ} \mathrm{C} .{ }^{1} \mathrm{H}$ NMR $(500 \mathrm{MHz}$, $\left.\mathrm{CDCl}_{3}\right): \delta=3.33\left(\mathrm{~s}, 3 \mathrm{H}, \mathrm{NCH}_{3}\right), 3.82\left(\mathrm{~s}, 3 \mathrm{H}, \mathrm{OCH}_{3}\right), 4.05\left(\mathrm{~s}, 2 \mathrm{H}, \mathrm{CH}_{2}\right)$, $6.81(\mathrm{~d}, J=8.1 \mathrm{~Hz}, 1$ arom. H), 6.92 (m, 1 arom. H), 7.08 (dd, $J=7.4$ 
$\mathrm{Hz}, 1.0 \mathrm{~Hz}, 1$ arom. H), $7.16\left(\mathrm{t}, J=8.2 \mathrm{~Hz}, 1\right.$ arom, H), $7.43\left(\mathrm{~s}, 1 \mathrm{H} \mathrm{CH}_{\mathrm{th}}\right) ;{ }^{13} \mathrm{C}$ NMR $(125.76$ $\mathrm{MHz}, \mathrm{CD}_{2} \mathrm{Cl}_{2}$ ): $\delta=28.50,36.80,51.84,112.16,114.77,120.34,127.50,129.84,131.40,133.27$, 134.50, 140.26, 153.34, 162.28.

Anal. Calcd for $\mathrm{C}_{14} \mathrm{H}_{13} \mathrm{NO}_{2} \mathrm{~S}$ (259.32): C 64.84, H 5.05, N 5.40; found C 64.48, H 5.01, N 5.67.

\section{Acknowledgements}

The author thanks Prof. Dr. Karl Leo, Director of the Institut für Angewandte Photophysik, TU Dresden, for his interest and generous technical support.

\section{References}

1. (a) Hartmann, H.; Scheithauer, S. J. Prakt. Chem. 1969, 311, 827 - 843. (b) Hartmann, H. J. Prakt. Chem. 1967, 36, 50 - 72.

2. Rogler, W.; Kanitz, A.; Hartmann, H.; Schumann, J. WO 200153286, 2001; Chem. Abstr. 135, 13739.

3. Hartmann, H.; Zug, I. J. Chem. Soc., Perkin. Trans. 1, 2000, 4316 - 4320.

4. Oliva, M. M.; Casado, J.; Raposo, M. M. M.; Fonesca, A. M. C.; Hartmann, H.; Hernández, V.; Navarrete, J. T. L. J. Org. Chem. 2006, 71, 7509 - 7520.

5. (a) Collado, D.; Casado, J.; Gonzales, S. R.; Navarrete, J. T. L.; Suau, R.; Perez-Inestrosa, E.; Pappenfus, T. M.; Raposo, M. M. M. Chem. Eur. J. 2011, 17, 498 - 507. (b) Keil, D.; Hartmann, H.; Reichardt, C. Liebigs Ann. Chem. 1993, 935 - 939.

6. Hartmann, H.; Eckert, K.; Schröder, A. Angew. Chem. Int. Ed. 2000, 39, 556 - 558.

7. (a) Tabet, A.; Schröder, A.; Hartmann, H.; Rohde, D.; Dunsch, L. Org. Lett. 2003, 5, 1817 1820. (b) Rohde, D.; Dunsch, L.; Tabet, A.; Hartmann, H.; Fabian, J. J. Phys. Chem. B, 2006, 110,8223 - 8231 .

8. Tabet, A.; Hartmann, H. Synthesis 2005, 610 - 616.

9. Rapta, P.; Tabet, A.; Hartmann, H.; Dunsch, L. J. Mater. Chem. 2007, 17, 4998 - 5007.

10. Kanitz, A.; Schumann, J.; Scheffel, M.; Rajoelson, S.; Rogler, W.; Hartmann, H.; Rohde, D. Chem. Lett. 2002, $896-897$.

11. (a) Watanabe, M.; Yamamaoto, T.; Nishiyama, M. Chem. Commun. 2000, 122 - 134. (b) Lu, Z.; Twieg, R. J. Tetrahedron 2005, 61, 903 - 918.

12. Bedworth, P. V.; Cai, Y.; Jen, A.; Marder, S. R. J. Org. Chem. 1996, 61, $2242-2246$.

13. (a) Heyde, C.; Zug, I.; Hartmann, H. Eur. J. Org. Chem. 2000, 3273 - 3278. (b) Eckert, K.; Schröder, A.; Hartmann, H. Eur. J. Org. Chem. 2000, 1327 - 1334.

14. Noack, A.; Hartmann, H. Tetrahedron 2002, 58, 2137 - 2146.

15. Hartmann, H. Z. Naturforsch. 2011, 66B, 711 - 714.

16. Hartmann, H.: Gerstner, P.; Rohde, D. Org. Lett. 2001, 3, 1673 - 1675. 
17. Svetkin, Y. V. Zh. Obsh. Khim. 1958, 28, $713-715$.

18. Cymerman-Craig, J.; Rogers, W. P.; Warwick, G. P. Austr. J. Chem. 1955, 8, 252 - 257.

19. Bennett, G. M.; Hafez, M. M. J. Chem. Soc. 1947, 287 - 288. 